\title{
KIT Mutation Analysis Was Not Performed
}

National Cancer Institute

\section{Source}

National Cancer Institute. KIT Mutation Analysis Was Not Performed. NCI Thesaurus.

Code C160391.

An indication that KIT mutation analysis was not performed during the study. 